\title{
NONLINEAR REPTATION IN MOLECULAR BASED HYSTERESIS MODELS FOR POLYMERS
}

\author{
BY \\ H. T. BANKS (Center for Research in Scientific Computation, North Carolina State University, \\ Raleigh, North Carolina 27695-8205).
}

NEGASH G. MEDHIN (Center for Research in Scientific Computation, North Carolina State University, Raleigh, North Carolina 27695-8205),

AND

\begin{abstract}
GABRIELLA A. PINTER (Department of Mathematical Sciences, University of Wisconsin-Milwaukee, Milwaukee, Wisconsin 53201-0413)
\end{abstract}

\begin{abstract}
We extend the linear "stick-slip" models of Doi-Edwards and JohnsonStacer to nonlinear tube reptation models. We then show that such models, when combined with probabilistic formulations allowing distributions of relaxation times, provide a good description of dynamic experiments with highly filled rubber in tensile deformations. A connection to other applications including dielectric polarization and reptation in other viscoelastic materials (e.g., living tissue) is noted.
\end{abstract}

1. Introduction. This note is prompted by several thrusts in our research efforts. The first is to extend linear reptation models for polymeric materials to models incorporating nonlinearities and to use the resulting systems to explain molecular based hysteresis (e.g., via internal variable formulations). A second direction involves exploration of multiscale aspects of polymeric structural modeling with uncertainty at the molecular (micro) level. We do this in the context of a probabilistic formulation of the models to produce a suitable overall system (macro) response to deformations. The ideas are illustrated in a specific application to highly filled rubber exhibiting significant hysteresis as well as nonlinearity in tensile and shear deformations as depicted in Figure 1.

Indeed, the ideas reported here were motivated by earlier efforts (summarized in [7] and the references therein) using a phenomenological approach to filled rubber modeling where we encountered significant hysteresis. The phenomenological approach entailed the use of Boltzmann hysteresis operators with nonlinearities that were necessary to describe experimental data. Our desire to understand the models at a more mechanistic

Received December 5, 2003.

2000 Mathematics Subject Classification. Primary 35R10, 35R30, 74D10, 74E35, 74H45.

Key words and phrases. viscoelastic, hysteresis, polymers, molecular models, relaxation times, probability distributions, uncertainty. 


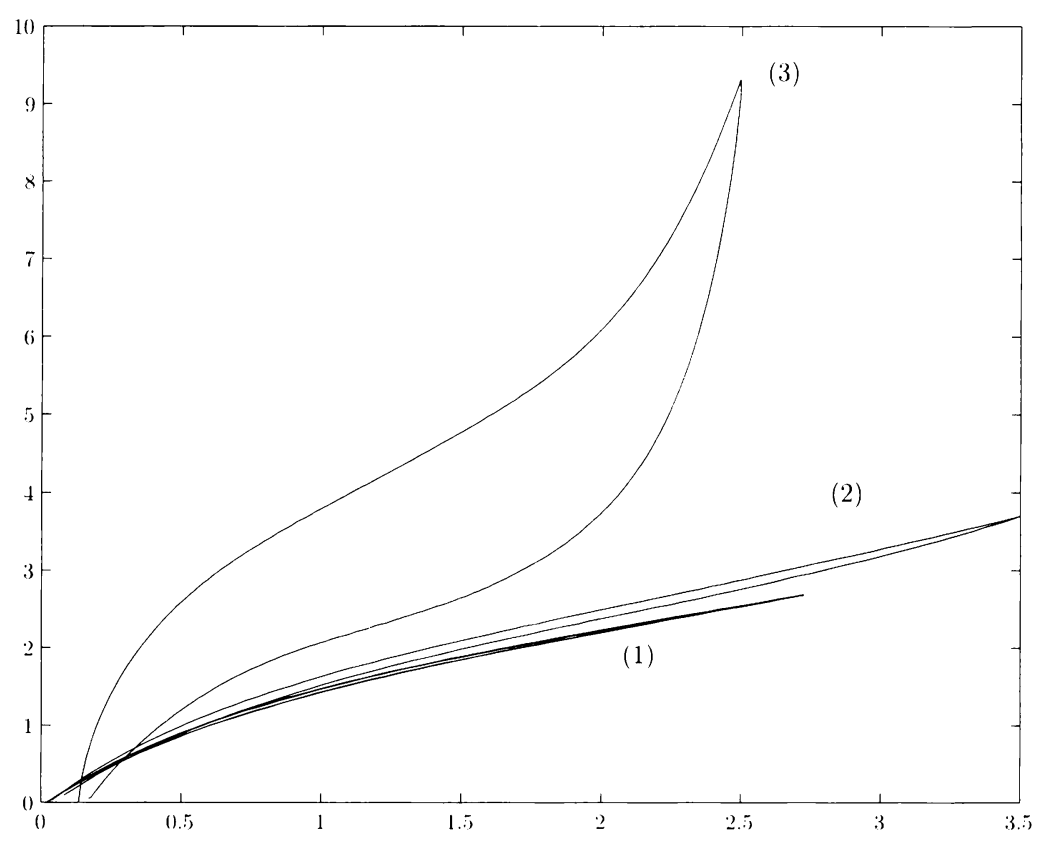

FIG. 1. Experimental stress-strain curves for (1) unfilled, (2) lightly filled and (3) highly filled rubber in tensile deformations

level led to the linear molecular models of Doi and Edwards [15] and Johnson and Stacer [20]. Linear versions of those formulations did not lead (see [6]) to the types of nonlinear hysteresis formulations sought after.

The focus here is on viscoelastic polymers, but the underlying ideas are much more widely applicable to problems in biology (living tissue. disease pathogenesis [5]), dielectric materials (polarization effects), industrial fluids (polymeric melts), and ecological migrations (hidden or internal episodic behaviors). For example, treating shear waves in living tissue requires nonlinear constitutive laws that are hysteretic in nature $([3,8,17])$. Molecular level (internal strain) formulations also utilize multiple relaxation time constants precisely such as those in the models developed below. Multiple relaxation times play an important role in molecular based (tube reptation) nonlinear constitutive models for the flow of polyethylene melts $([11,12,13,18,22])$. For dielectric materials, it has been known for some time $([14,25,26])$ that one needs distributed relaxation times in polarization models for heterogeneous materials. For example, it is desirable to represent the complex dielectric permittivity $([14])$ in terms of a probability density $\phi$ over relaxation times as in

$$
\varepsilon(\omega)=\varepsilon_{\infty}+\left(\varepsilon_{s}-\varepsilon_{\infty}\right) \int_{0}^{\infty} \frac{\phi(\tau) d \tau}{1+j \omega \tau} .
$$

This can be written in a more familiar form as

$$
\varepsilon(\omega)=\varepsilon_{\infty}+\left(\varepsilon_{s}-\varepsilon_{\infty}\right) \int_{0}^{\infty} \frac{\phi(\tau) d \tau}{1+\omega^{2} \tau^{2}}-j\left(\varepsilon_{s}-\varepsilon_{\infty}\right) \int_{0}^{\infty} \frac{\omega \tau \phi(\tau) d \tau}{1+\omega^{2} \tau^{2}} .
$$


The latter formulations are analogous to proposed forms of the "elastic" moduli based on experimental observations in the works of Ferry, Andrews, Ter Haar and others [2, $16,23,24,27]$. We describe in detail the incorporation of multiple relaxation times in our models in Sec. 4.

In Sec. 2 we give a brief review of the linear reptation models developed by Johnson and Stacer and then in the next section provide the details on our extension to nonlinear versions of these models. In Secs. 4 and 5 we explain how these models have led to a molecular based fit of dynamic models to experimental data for highly filled rubber in tensile and shear deformations.

2. Linear reptation models. Tube reptation models for deformations of viscoelastic polymers were introduced by Doi and Edwards [15] and were further developed by Johnson, et.al., in several papers $[19,20]$. In this section we give a brief overview of their models and assumptions in the case of tensile deformations since they serve as a starting point for the derivation of our nonlinear reptation model in Sec. 3.

The Doi-Edwards "stick-slip" model assumes that the polymer is composed of chemically cross-linked (CC) tubes that contain physically constrained (PC) molecules. At the time an instantaneous tensile step-strain is applied, the entrapped PC molecules stick to the tube and elongate with it, but then they contract and slip back close to their original length. This provides the viscoelastic character of the material. In particular, the relaxation is modelled in the following way. Let $L(t)$ denote the length of the chemically cross-linked molecule, while $\ell(t)$ stands for the length of the physically constrained one. Assume that the PC molecule elongates to length $\ell^{*}$ due to the applied step-strain. Then

$$
\ell(t)=\ell(0)+\left(\ell^{*}-\ell(0)\right) e^{-t / \tau} \quad 0=t_{0}<t<t_{1},
$$

where $\tau$ is the relaxation time for the "slip" motion and $t_{1}$ denotes the time the next step-strain is applied. It is assumed in [15] and in the continuum realization of this model in [20] that the "stick" phase of the motion at the time of the step-strain deforms the $\mathrm{PC}$ molecule proportionally with the $\mathrm{CC}$ tube deformation, i.e.,

$$
\frac{\Delta \ell_{i}}{\Delta L_{i}}=\frac{\ell_{i}}{L_{i}}, \quad i=0,1, \ldots
$$

Here $L_{i}$ and $\ell_{i}$ denote the length of the CC and PC molecules at time $t_{i}$, respectively, while $\Delta L_{i}$ and $\Delta \ell_{i}$ stand for the instantaneous stretches, i.e., $L_{i}=L_{i-1}+\Delta L_{i-1}, \ell_{i}=$ $\ell_{i-1}+\Delta \ell_{i-1}$. Using this assumption we can write (2.1) as

$$
\ell(t)=\ell_{0}+\frac{\ell_{0}}{L_{0}} \Delta L_{0} e^{-t / \tau}, \quad 0=t_{0}<t<t_{1} .
$$

Continuous motion is approximated by a series of step-strain deformations applied in $\Delta t$ intervals, where $\Delta t \rightarrow 0$. This leads to the relaxation equation

$$
\ell(t)=\ell_{0}+\int_{0}^{t} \frac{\ell(s)}{L(s)} \frac{d L(s)}{d s} e^{-(t-s) / \tau} d s
$$

or, in differential form,

$$
\frac{d \ell}{d t}=\frac{\ell_{0}}{\tau}-\left(\frac{1}{\tau}-\frac{1}{L} \frac{d L}{d t}\right) \ell .
$$


When this model is assembled into a 3-D continuum model of a solid, one defines a unit box or cell at each material point oriented by the principal stretches $\left(\lambda_{1 c}, \lambda_{2 c}, \lambda_{3 c}\right)$ of the $\mathrm{CC}$ system with an inside box with parallel sides $\left(\lambda_{1 p}, \lambda_{2 p}, \lambda_{3 p}\right)$ for the PC system. Stresses are calculated by determining how the strain energy function $W$, which has contribution $W_{c c}$ from the CC box and $W_{p c}$ from the $\mathrm{PC}$ system, changes with respect to the applied stretches or displacements of the CC system. In turn, the stretches of the $\mathrm{PC}$ box are treated as internal variables depending on the stretches of the CC system. We note that as an analogy to (2.2) we have

$$
\frac{\partial \lambda_{j p}}{\partial \lambda_{i c}}=\frac{\lambda_{j p}}{\lambda_{i c}} \delta_{j i}
$$

where $\delta_{j i}$ is the Kronecker delta function. Thus we have that the strain energy density function is

$$
W=W_{p c}+W_{c c}=W_{p c}\left(\lambda_{1 p}, \lambda_{2 p}, \lambda_{3 p}\right)+W_{c c}\left(\lambda_{1 c}, \lambda_{2 c}, \lambda_{3 c}\right),
$$

and the internal dynamics (2.4) (with $\ell_{0}=1$ ) yields

$$
\frac{d \lambda_{i p}}{d t}=\frac{1}{\tau}-\left(\frac{1}{\tau}-\frac{1}{\lambda_{i c}} \frac{d \lambda_{i c}}{d t}\right) \lambda_{i p}
$$

since the principal directions of the PC and CC system are aligned. The Cauchy stress in the principal direction $e_{j}$ (where $e_{j}$ is a unit vector in the $x_{j}$ direction) is given by

$$
\tau_{j}=\lambda_{j c} \frac{\partial W}{\partial \lambda_{j c}}-P,
$$

where $P$ is the hydrostatic stress. We combine (2.6) and (2.8) with the assumption that $\lambda_{i p}$ depend on $\lambda_{i c}$ as internal variables and obtain

$$
\begin{aligned}
\tau_{j} & =\lambda_{j c} \frac{\partial W_{c c}}{\partial \lambda_{j c}}+\lambda_{j c} \sum_{i=1}^{3} \frac{\partial W_{p c}}{\partial \lambda_{i p}} \frac{\partial \lambda_{i p}}{\partial \lambda_{j c}}-P \\
& =\lambda_{j c} \frac{\partial W_{c c}}{\partial \lambda_{j c}}+\lambda_{j p} \frac{\partial W_{p c}}{\partial \lambda_{j p}}-P
\end{aligned}
$$

by applying (2.5). If we choose $j=1$ for the direction of loading, we have

$$
\tau_{2}=\tau_{3}=0=\lambda_{2 c} \frac{\partial W_{c c}}{\partial \lambda_{2 c}}+\lambda_{2 p} \frac{\partial W_{p c}}{\partial \lambda_{2 p}}-P
$$

because stresses are zero on the sides of a tensile sample. Since $P$ can be determined from (2.9), we obtain that the tensile Cauchy stress is

$$
\tau_{1}=\lambda_{1 c}\left(\frac{\partial W_{c c}}{\partial \lambda_{1 c}}-\lambda_{2 c} \frac{\partial W_{c c}}{\partial \lambda_{2 c}}\right)+\lambda_{1 p}\left(\frac{\partial W_{p c}}{\partial \lambda_{1 p}}-\lambda_{2 p} \frac{\partial W_{p c}}{\partial \lambda_{2 p}}\right),
$$

while the engineering stress is given by

$$
\sigma_{1}=\frac{\tau_{1}}{\lambda_{1 c}}=\left(\frac{\partial W_{c c}}{\partial \lambda_{1 c}}-\lambda_{2 c} \frac{\partial W_{c c}}{\partial \lambda_{2 c}}\right)+\frac{\lambda_{1 p}}{\lambda_{1 c}}\left(\frac{\partial W_{p c}}{\partial \lambda_{1 p}}-\lambda_{2 p} \frac{\partial W_{p c}}{\partial \lambda_{2 p}}\right) .
$$

This model was analyzed in [6] for incompressible rubber materials undergoing large dynamic tensile strains with a particular strain energy function provided in [21]. 
Let $u_{p}, u_{c}$ denote the deformation of the $\mathrm{PC}$ and $\mathrm{CC}$ boxes, respectively. With $\lambda_{1 p}=$ $1+\partial_{x} u_{p}$ and $\lambda_{1 c}=1+\partial_{x} u_{c},(2.7)$ implies that

$$
\frac{\partial}{\partial t}\left(1+\partial_{x} u_{p}\right)=\frac{1}{\tau}-\left\{\frac{1}{\tau}-\left(1+\partial_{x} u_{c}\right)^{-1} \frac{\partial^{2} u_{c}}{\partial t \partial x}\right\}\left(1+\partial_{x} u_{p}\right) .
$$

This in turn yields the internal dynamics

$$
\dot{\varepsilon}_{1}+\frac{1}{\tau} \varepsilon_{1}=\dot{\varepsilon}\left(1+\varepsilon_{1}\right) /(1+\varepsilon)
$$

in terms of the infinitesimal strains $\varepsilon_{1}=\partial_{x} u_{p}$ for the PC system and $\varepsilon=\partial_{x} u_{c}$ for the $\mathrm{CC}$ system. It is shown in [6] that the above derivation leads to an approximation of the engineering stress in the form

$$
\sigma_{1}\left(\varepsilon, \varepsilon_{1}\right) \approx\left(1248-1014 \frac{\partial u_{p}}{\partial x}\right) \frac{\partial u_{c}}{\partial x}+1014 \frac{\partial u_{p}}{\partial x}=\left(1248-1014 \varepsilon_{1}\right) \varepsilon+1014 \varepsilon_{1} .
$$

This can be combined with the basic model for the longitudinal vibrations of a rubber rod as given, for example in [10], by

$$
\rho A_{c} \frac{\partial^{2} u_{c}}{\partial t^{2}}-A_{c} C_{D} \frac{\partial^{3} u_{c}}{\partial t \partial x^{2}}-\frac{\partial}{\partial x}\left[A_{c} \sigma_{1}\right]=F,
$$

where $F$ is the applied external force, $A_{c}$ is the cross-sectional area and $\rho$ is the mass density of the material.

3. Nonlinear reptation model. In this section we present a nonlinear extension of the "stick-slip" model of Doi and Edwards for tensile deformations. A crucial assumption throughout the derivation in the previous section was that the elongation of the PC molecules is proportional to that of the CC molecules during the "stick" phase of the motion, i.e., $\frac{\Delta \ell}{\Delta L}=\frac{\ell}{L}$, or $\frac{\partial \lambda_{j p}}{\partial \lambda_{i c}}=\frac{\lambda_{j p}}{\lambda_{i c}} \delta_{j i}$ as in (2.2) and (2.5). It might be expected that for certain materials and large strains this relationship is not strictly linear, but rather, it is described by a general nonlinear function $f$ that may also depend on time, i.e.,

$$
\frac{\Delta \ell}{\Delta L}=f\left(t, \frac{\ell}{L}\right), \text { or } \frac{\partial \lambda_{j p}}{\partial \lambda_{i c}}=f\left(t, \frac{\lambda_{j p}}{\lambda_{i c}}\right) \delta_{j i} .
$$

Thus we have that the relaxation equation after an instantaneous step-strain, in contrast with $(2.3)$, is given by

$$
\ell(t)=\ell_{0}+f\left(t_{0}, \frac{\ell_{0}}{L_{0}}\right) \Delta L_{0} e^{-\left(t-t_{0}\right) / \tau}, \quad 0=t_{0}<t<t_{1} .
$$

Similarly, for $t \in\left(t_{m-1}, t_{m}\right), m \geq 1$ we have

$$
\ell(t)=\ell_{0}+\sum_{i=0}^{m-1} f\left(t_{i}, \frac{\ell_{i}}{L_{i}}\right) \Delta L_{i} e^{-\left(t-t_{i}\right) / \tau} .
$$

In the limit, as $\Delta t=t_{m}-t_{m-1}$ tends to zero we obtain

$$
\ell(t)=\ell_{0}+\int_{0}^{t} f\left(s, \frac{\ell(s)}{L(s)}\right) \frac{d L(s)}{d s} e^{-(t-s) / \tau} d s .
$$

Thus

$$
\frac{d \ell}{d t}=f\left(t, \frac{\ell(t)}{L(t)}\right) \frac{d L}{d t}-\frac{1}{\tau}\left(\ell(t)-\ell_{0}\right)
$$


describes the continuous motion of the CC-PC system in differential form. Now we take $\ell_{0}=1, \ell=1+\varepsilon_{1}, L=1+\varepsilon$ and we obtain the internal dynamics

$$
\dot{\varepsilon}_{1}+\frac{1}{\tau} \varepsilon_{1}=\dot{\varepsilon} f\left(t, \frac{1+\varepsilon_{1}}{1+\varepsilon}\right) .
$$

As in the previous case we can add this equation to the general model for the longitudinal vibrations of a rubber rod (2.10).

Before turning to the engineering stress $\sigma_{1}$ and describing how it is affected by the nonlinear assumption (3.1), we consider approximations of (3.2). Assuming that $f$ is time invariant and expanding it in a Taylor series, we obtain

$$
\begin{aligned}
f\left(\frac{1+\varepsilon_{1}}{1+\varepsilon}\right) & =f(1)+f^{\prime}(1)\left(\frac{1+\varepsilon_{1}}{1+\varepsilon}-1\right)+\frac{f^{\prime \prime}(1)}{2}\left(\frac{1+\varepsilon_{1}}{1+\varepsilon}-1\right)^{2}+\ldots \\
& =f(1)+f^{\prime}(1)\left(\frac{\varepsilon_{1}-\varepsilon}{1+\varepsilon}\right)+\frac{f^{\prime \prime}(1)}{2}\left(\frac{\varepsilon_{1}-\varepsilon}{1+\varepsilon}\right)^{2}+\ldots \\
& =\gamma_{0}+\delta \varepsilon_{1}+\gamma_{1} \varepsilon+\gamma_{2} \varepsilon^{2}+\text { h.o.t. }\left(\varepsilon^{k}, k \geq 3\right)+\text { h.o.t. }\left(\varepsilon_{1}^{k}, k \geq 2\right) \\
& + \text { h.o.t. }\left(\varepsilon^{k} \varepsilon_{1}^{j}, k, j \geq 1\right) .
\end{aligned}
$$

If $\varepsilon_{1}<<\varepsilon$, we find the approximate equation for (3.2) given by

$$
\dot{\varepsilon}_{1}+\frac{1}{\tau} \varepsilon_{1}=\dot{\varepsilon}\left(\gamma_{0}+\gamma_{1} \varepsilon+\gamma_{2} \varepsilon^{2}\right)
$$

We note that this approximation corresponds to our earlier phenomenological internal variable formulation in $[10]$

where $g_{v}$ is a cubic polynomial.

$$
\dot{\varepsilon}_{1}+\frac{1}{\tau} \varepsilon_{1}=\frac{d}{d t} g_{v}(\varepsilon)
$$

Now we turn to the derivation of an approximation to the engineering stress $\sigma_{1}$ in presence of the nonlinear assumption (3.1). Let us make similar assumptions to those that we made for the linear model in Sec. 2. Namely, we have that the strain energy function $W$ is given as

$$
W=W_{p c}+W_{c c}=W_{p c}\left(\lambda_{1 p}, \lambda_{2 p}, \lambda_{3 p}\right)+W_{c c}\left(\lambda_{1 c}, \lambda_{2 c}, \lambda_{3 c}\right),
$$

so that

$$
\tau_{j}=\lambda_{j c} \frac{\partial W}{\partial \lambda_{j c}}-P,
$$

where the $\lambda_{i p}$ and $\lambda_{i c}$ are the principal stretches of the PC and CC systems, respectively. Additionally, we assume that $f$ in (3.1) is independent of time, i.e., $f(t, \theta)=f(\theta)$. Since the $\lambda_{i p}$ depend on the $\lambda_{i c}$ as internal variables, the Cauchy stress can again be given in the form

$$
\tau_{j}=\lambda_{j c} \frac{\partial W_{c c}}{\partial \lambda_{j c}}+\lambda_{j c} \sum_{i=1}^{3} \frac{\partial W_{p c}}{\partial \lambda_{i p}} \frac{\partial \lambda_{i p}}{\partial \lambda_{j c}}-P .
$$

At this point our derivation differs from the one in the previous section. By (3.1)

$$
\frac{\partial \lambda_{i p}}{\partial \lambda_{j c}}=f\left(\frac{\lambda_{i p}}{\lambda_{j c}}\right) \delta_{i j}
$$


so (3.5) yields

$$
\tau_{j}=\lambda_{j c} \frac{\partial W_{c c}}{\partial \lambda_{j c}}+\lambda_{j c} \sum_{i=1}^{3} \frac{\partial W_{p c}}{\partial \lambda_{i p}} f\left(\frac{\lambda_{i p}}{\lambda_{j c}}\right) \delta_{i j}-P .
$$

If $j=1$ denotes the direction of loading, then we have

$$
\tau_{2}=\tau_{3}=0=\lambda_{2 c} \frac{\partial W_{c c}}{\partial \lambda_{2 c}}+\lambda_{2 c} \frac{\partial W_{p c}}{\partial \lambda_{2 p}} f\left(\frac{\lambda_{2 p}}{\lambda_{2 c}}\right)-P .
$$

We can express $P$ from (3.7) and substitute into (3.6) with $j=1$ to find

$$
\tau_{1}=\lambda_{1 c} \frac{\partial W_{c c}}{\partial \lambda_{1 c}}+\lambda_{1 c} \frac{\partial W_{p c}}{\partial \lambda_{1 p}} f\left(\frac{\lambda_{1 p}}{\lambda_{1 c}}\right)-\left[\lambda_{2 c} \frac{\partial W_{c c}}{\partial \lambda_{2 c}}+\lambda_{2 c} \frac{\partial W_{p c}}{\partial \lambda_{2 p}} f\left(\frac{\lambda_{2 p}}{\lambda_{2 c}}\right)\right] .
$$

Now the engineering stress $\sigma_{1}$ is given by

$$
\sigma_{1}=\frac{\partial W_{c c}}{\partial \lambda_{1 c}}-\frac{\lambda_{2 c}}{\lambda_{1 c}} \frac{\partial W_{c c}}{\partial \lambda_{2 c}}+\frac{\partial W_{p c}}{\partial \lambda_{1 p}} f\left(\frac{\lambda_{1 p}}{\lambda_{1 c}}\right)-\frac{\lambda_{2 c}}{\lambda_{1 c}} \frac{\partial W_{p c}}{\partial \lambda_{2 p}} f\left(\frac{\lambda_{2 p}}{\lambda_{2 c}}\right) .
$$

The first two terms on the right side above constitute the contribution of $W_{c c}$ to the engineering stress $\sigma_{1}$, while the last two terms provide the contribution of $W_{p c}$. We assume that the energy density function $W$ is given as in (3.4) with

$$
\begin{gathered}
W_{c c}=C_{1}\left(I_{1}-3\right)+C_{2}\left(I_{2}-3\right), \\
W_{p c}=C_{3}\left(\tilde{I}_{1}-3\right)+C_{4}\left(\tilde{I}_{1}-3\right)^{2}+C_{5}\left(\tilde{I}_{2}-3\right)^{3},
\end{gathered}
$$

where specific values of the coefficients $C_{1}, \ldots, C_{5}$ can be chosen as in [6] given the strain energy function suggested by Johnson and Stacer based on experimental data [21]. Here the strain invariants are

$$
I_{1}=\lambda_{1 c}^{2}+\lambda_{2 c}^{2}+\lambda_{3 c}^{2}, \quad I_{2}=\lambda_{1 c}^{2} \lambda_{2 c}^{2}+\lambda_{1 c}^{2} \lambda_{3 c}^{2}+\lambda_{2 c}^{2} \lambda_{3 c}^{2},
$$

while

$$
\tilde{I}_{1}=\lambda_{1 p}^{2}+\lambda_{2 p}^{2}+\lambda_{3 p}^{2}, \quad \tilde{I}_{2}=\lambda_{1 p}^{2} \lambda_{2 p}^{2}+\lambda_{1 p}^{2} \lambda_{3 p}^{2}+\lambda_{2 p}^{2} \lambda_{3 p}^{2}
$$

We also impose the incompressibility condition in the principle stretches for the PC and CC systems, respectively, i.e., $\lambda_{1 p} \lambda_{2 p} \lambda_{3 p}=\lambda_{1 c} \lambda_{2 c} \lambda_{3 c}=1$. We can compute the contribution of $W_{c c}$ to the engineering stress exactly the same way as in [6]:

$$
\begin{aligned}
& \frac{\partial W_{c c}}{\partial \lambda_{1 c}}-\frac{\lambda_{2 c}}{\lambda_{1 c}} \frac{\partial W_{c c}}{\partial \lambda_{2 c}}=A \lambda_{1 c}+B-\frac{A}{\lambda_{1 c}^{2}}-\frac{B}{\lambda_{1 c}^{3}} \\
& =A(1+\varepsilon)+B-\frac{A}{(1+\varepsilon)^{2}}-\frac{B}{(1+\varepsilon)^{3}},
\end{aligned}
$$

where $A$ and $B$ are appropriate constants depending on the values of $C_{1}, \ldots, C_{5}$. Note that in this calculation we use the relationship $\lambda_{2 c}=\lambda_{3 c}=\frac{1}{\sqrt{\lambda_{1 c}}}$ in the incompressible CC system under tensile deformation in the $x_{1}$ direction. A Taylor expansion of the negative powers of $(1+\varepsilon)$ in (3.9) yields

$$
\frac{\partial W_{c c}}{\partial \lambda_{1 c}}-\frac{\lambda_{2 c}}{\lambda_{1 c}} \frac{\partial W_{c c}}{\partial \lambda_{2 c}}=a_{1} \varepsilon+a_{2} \varepsilon^{2}+\cdots+a_{k} \varepsilon^{k}+\ldots
$$


Next we turn to the contribution of $W_{p c}$ to the engincering stress, where the nonlinear assumption (3.1) modifies the results of the linear case. We find that

$$
\begin{aligned}
& f\left(\frac{\lambda_{1 p}}{\lambda_{1 c}}\right) \frac{\partial W_{p c}}{\partial \lambda_{1 p}}-\frac{\lambda_{2 c}}{\lambda_{1 c}} f\left(\frac{\lambda_{2 p}}{\lambda_{2 c}}\right) \frac{\partial W_{p c}}{\partial \lambda_{2 p}} \\
& =f\left(\frac{\lambda_{1 p}}{\lambda_{1 c}}\right)\left[2 C_{3} \lambda_{1 p}+4 C_{4} \lambda_{1 p}\left(\lambda_{1 p}^{2}+\frac{2}{\lambda_{1 p}}-3\right)+12 C_{5}\left(2 \lambda_{1 p}+\frac{1}{\lambda_{1 p}^{2}}-3\right)^{2}\right] \\
& -\frac{1}{\lambda_{1 c}^{3 / 2}} f\left(\sqrt{\frac{\lambda_{1 c}}{\lambda_{1 p}}}\right)\left[2 C_{3} \frac{1}{\sqrt{\lambda_{1 p}}}+4 C_{4} \frac{1}{\sqrt{\lambda_{1 p}}}\left(\lambda_{1 p}^{2}+\frac{2}{\lambda_{1 p}}-3\right)\right. \\
& \left.+3 C_{5}\left(2 \lambda_{1 p}+\frac{1}{\lambda_{1 p}^{2}}-3\right)^{2}\left(2 \lambda_{1 p}^{3 / 2}+\frac{2}{\lambda_{1 p}^{3 / 2}}\right)\right] .
\end{aligned}
$$

We assume that $\varepsilon_{1}<<\varepsilon$ and use the following approximations:

$$
\begin{aligned}
f\left(\frac{\lambda_{1 p}}{\lambda_{1 c}}\right)= & f\left(\frac{1+\varepsilon_{1}}{1+\varepsilon}\right)=f(1)+f^{\prime}(1) \frac{\varepsilon_{1}-\varepsilon}{1+\varepsilon}+\frac{f^{\prime \prime}(1)}{2}\left(\frac{\varepsilon_{1}-\varepsilon}{1+\varepsilon}\right)^{2}+\ldots \\
\approx & f(1)+f^{\prime}(1) \frac{\varepsilon_{1}-\varepsilon}{1+\varepsilon} \approx f(1)+f^{\prime}(1)(-\varepsilon)\left(1-\varepsilon+\varepsilon^{2}-\varepsilon^{3}+\ldots\right) \\
= & \alpha_{0}+\alpha_{1} \varepsilon+\alpha_{2} \varepsilon^{2}+\ldots, \\
f\left(\sqrt{\left.\frac{\lambda_{1 c}}{\lambda_{1 p}}\right)=}\right. & f\left(\sqrt{\frac{1+\varepsilon}{1+\varepsilon_{1}}}\right) \approx f(\sqrt{1+\varepsilon})=f(1)+f^{\prime}(1)(\sqrt{1+\varepsilon}-1) \\
& \quad+\frac{f^{\prime \prime}(1)}{2}(\sqrt{1+\varepsilon}-1)^{2}+\ldots=\beta_{0}+\beta_{1} \varepsilon+\beta_{2} \varepsilon^{2}+\beta_{3} \varepsilon^{3}+\ldots,
\end{aligned}
$$

where we expanded $\sqrt{1+\varepsilon}$ in powers of $\varepsilon$. With similar expansions for $\frac{1}{\lambda_{1 p}}, \frac{1}{\lambda_{1 p}^{2}}, \frac{1}{\sqrt{\lambda_{1 p}}}$, $\lambda_{1 p}^{3 / 2}, \lambda_{1 p}^{-3 / 2}, \lambda_{1 c}^{-3 / 2}$, we obtain that

$$
\begin{aligned}
& f\left(\frac{\lambda_{1 p}}{\lambda_{1 c}}\right) \frac{\partial W_{p c}}{\partial \lambda_{1 p}}-\frac{\lambda_{2 c}}{\lambda_{1 c}} f\left(\frac{\lambda_{2 p}}{\lambda_{2 c}}\right) \frac{\partial W_{p c}}{\partial \lambda_{2 p}}=\delta_{1} \varepsilon_{1}+\delta_{2} \varepsilon_{1}^{2}+\cdots+\gamma_{1} \varepsilon+\gamma_{2} \varepsilon^{2}+\ldots \\
& + \text { h.o.t. }\left(\varepsilon^{k} \varepsilon_{1}^{j}, k, j \geq 1\right) .
\end{aligned}
$$

Combining (3.10) and (3.11) with (3.8), we have the following approximation for the engineering stress:

$$
\begin{aligned}
& \sigma_{1}\left(\varepsilon, \varepsilon_{1}\right)=c_{1} \varepsilon+c_{2} \varepsilon^{2}+c_{3} \varepsilon^{3}+\text { h.o.t. }\left(\varepsilon^{k}, k \geq 4\right)+\delta_{1} \varepsilon_{1}+\text { h.o.t. }\left(\varepsilon_{1}^{k}, k \geq 2\right) \\
& + \text { h.o.t. }\left(\varepsilon^{k} \varepsilon_{1}^{j}, k, j \geq 1\right) \approx c_{1} \varepsilon+c_{2} \varepsilon^{2}+c_{3} \varepsilon^{3}+\delta_{1} \varepsilon_{1} .
\end{aligned}
$$

We remark that the constitutive equation $\sigma_{1}=g_{e}(\varepsilon)+C_{D} \dot{\varepsilon}+\mu_{1} \varepsilon_{1}$, where $g_{e}$ is a cubic polynomial of the form

$$
g_{e}(\varepsilon)=c_{1} \varepsilon+c_{2} \varepsilon^{2}+c_{3} \varepsilon^{3}
$$

was assumed based on phenomenological arguments in [10] and, as we shall note below in Sec. 5, it has been successfully used in reproducing experimental data for shear deformations of rubber samples as well as for large tensile deformations of a rubber rod. We also point out that the presence of the $\dot{\varepsilon}$ term in this formulation for $\sigma_{1}$ represents 
an approximation to a damping mechanism (certainly present in the case of highly filled rubber) and does not result from the nonlinear reptation formulation.

4. General tensile models with multiple relaxation times. The above model with (2.10), (3.2) (with $f$ independent of $t$ ) and (3.12) forms the basis of a general class of nonlinear deformation models for polymers in tension. More generally, the system written in distributional or generalized sense (see $[7,10])$ has the form

$$
\rho A_{c} \frac{\partial^{2} u}{\partial t^{2}}-\frac{\partial}{\partial x}\left(A_{c} \Sigma_{1}\left(\varepsilon, \dot{\varepsilon}, \varepsilon_{1}\right)\right)=F
$$

where

$$
\Sigma_{1}\left(\varepsilon, \dot{\varepsilon}, \varepsilon_{1}\right)=\sigma_{\text {elast }}(\varepsilon, \dot{\varepsilon})+\sigma_{\text {visco }}\left(\varepsilon_{1}\right)
$$

and

$$
\dot{\varepsilon}_{1}+\frac{1}{\tau} \varepsilon_{1}=\dot{\varepsilon} f\left(\frac{1+\varepsilon_{1}}{1+\varepsilon}\right) .
$$

However, such models are based on the tacit assumption that all molecules relax with the same relaxation time $\tau$. There is substantial experimental evidence $[2,16,23,24,27]$ to suggest that the assumption of a uniform relaxation time is not valid. Indeed, when fitting "elastic" moduli

$$
E(\omega)=E^{\prime}(\omega)+j E^{\prime \prime}(\omega)=\int_{0}^{\infty} \frac{\omega^{2} \tau^{2}}{1+\omega^{2} \tau^{2}} \phi(\tau) d \tau+j \int_{0}^{\infty} \frac{\omega \tau}{1+\omega^{2} \tau^{2}} \phi(\tau) d \tau
$$

to response data in the frequency domain, it is often necessary to use a probability density $\phi$ for the distribution of relaxation times.

In the context of the reptation models developed here, this implies that one should replace the engineering stress $\Sigma_{1}$ in (4.2) by a probability measure or probability distribution dependent stress-strain law of the form

$$
\Sigma_{1}(\varepsilon, \dot{\varepsilon}, \Phi)=\sigma_{\text {elast }}(\varepsilon, \dot{\varepsilon})+\int_{\tau_{0}}^{\infty} \varepsilon_{1}(\tau) d \Phi(\tau),
$$

where $t \rightarrow \varepsilon_{1}(t ; \tau)$ is the solution of (4.3) for a given $\tau, \Phi$ is a probability distribution for the relaxation times $\tau$, and $\tau_{0}>0$ is a lower bound on possible relaxation times. For the special case of an absolutely continuous distribution this, of course, reduces to

$$
\Sigma_{1}(\varepsilon, \dot{\varepsilon}, \Phi)=\sigma_{\text {elast }}(\varepsilon, \dot{\varepsilon})+\int_{\tau_{0}}^{\infty} \varepsilon_{1}(\tau) \phi(\tau) d \tau,
$$

where $\phi=\Phi^{\prime}$. For a distribution consisting of a finite number of Dirac measures with atoms at $\tau_{1}, \tau_{2}, \ldots, \tau_{M}$, respectively, we have

$$
\Sigma_{1}(\varepsilon, \dot{\varepsilon}, \Phi)=\sigma_{\text {elast }}(\varepsilon, \dot{\varepsilon})+\sum_{i=1}^{M} p_{i} \varepsilon_{1}\left(\tau_{i}\right),
$$

where $\sum_{i=1}^{M I} p_{i}=1$ and $t \rightarrow \varepsilon_{1}\left(t ; \tau_{i}\right)$ satisfies (4.3) with $\tau=\tau_{i}, \quad i=1,2, \ldots, M$.

A general theory of existence and uniqueness that applies to systems (4.1) with various approximations of (4.3), and (4.5), i.e., the discrete measure case, can be found in [1]. A theory for continuous dependence of solutions with respect to parameters (specifically 
with respect to the discrete measure in (4.5)) can be pursued in the context of the Prohorov metric topology on the space of probability distributions. In this situation this is equivalent to the weak-star topology on the distributions if they are viewed as a subset of the dual space of $C\left[\tau_{0}, \bar{\tau}\right]$, the space of bounded continuous functions on a finite interval $\left[\tau_{0}, \bar{\tau}\right]$ of relaxation times (see $[4,5]$ for a discussion of the Prohorov metric and its use in inverse problems for systems containing uncertainty in the "parameters" in the setting of measure or probability distribution dependent systems). Efforts are currently underway to develop a rigorous theory for well-posedness (including continuous dependence with respect to the measures $\Phi$ ) of systems with (4.4) as the general measure dependent engineering stress law.

5. Application to experimental data. Systems such as (4.1), (4.3) with (4.4) certainly pose conceptual, theoretical and computational challenges even when treating forward or simulation problems. Even more difficulties are presented by estimation or inverse problems wherein one attempts to use experimental data to determine the distribution $\Phi$, parameters in $\sigma_{\text {elast }}$, or $f$ in (4.4) and (4.3), respectively. However, significant progress on such problems has been achieved. In a recent summary [7], results are reported on using $\Sigma_{1}$ of the form (4.5) in experimental, computational and theoretical investigations for filled rubber rods in tensile and shear deformations. In the following we describe the use of models with approximations such as in (3.3) and (3.12), that is, dropping the h.o.t. terms, to fit data from dynamic experiments for rubber samples in tension.

We summarize here some of the results obtained using dynamic experiments with a rubber rod with a tip mass at one end in uniaxial tensile deformation. Similar experimental efforts to validate models for filled rubber samples in shear were successfully carried out as well (see [7]). The experimental device constructed specifically for these tensile validation tests at the Thomas Lord Research Center of the Lord Corporation is depicted schematically in Figure 2.

This experiment produced data $\left\{z_{i}\right\}$ consisting of time measurements of force (at the tip of the rod, $x=0$ ) collected by a load cell. The data corresponds to the engineering stress $\Sigma_{1}$ at times $t_{i}$ at the top of the rod, $x=0$, multiplied by the cross sectional area $A_{c}$. Thus a least squares formulation for estimation of parameters $q$ has the form

$$
J(q)=\sum_{i}\left|z_{i}-A_{c} \Sigma_{1}\left(\varepsilon\left(t_{i}, 0\right), \dot{\varepsilon}\left(t_{i}, 0\right), \Phi\right)\right|^{2},
$$

with $\varepsilon(t, x)=\frac{\partial u}{\partial x}(t, x)$, where $\Sigma_{1}$ is given by (4.5). Here $\varepsilon_{1}$ satisfies (4.3) with $f$ approximated as in (3.3) for each $\tau_{i}$, and $\sigma_{\text {elast }}$ is given by

$$
\sigma_{\text {elast }}(\varepsilon, \dot{\varepsilon})=g_{e}(\varepsilon)+C_{D} \dot{\varepsilon},
$$

where $g_{e}$ is a cubic polynomial as in (3.13). To be more precise, experimental data suggested that the nonlinearity $f$ was not the same when tensile deformation was increasing as when decreasing. Hence the approximation in (3.3) was employed with two sets of $\gamma_{i}$ 's, one set $\left\{\gamma_{i}^{i n c}\right\}$ for increasing deformations and one set $\left\{\gamma_{i}^{\text {dec }}\right\}$ for decreasing deformations. The parameter $q$ to be estimated from the data using (5.1) thus consisted of $\rho, C_{D},\left\{\gamma_{i}^{i n c}\right\},\left\{\gamma_{i}^{\text {dec }}\right\}, c_{1}, c_{2}, c_{3}$ and the $\tau_{i}$ 's in (4.5). For the results described here we 


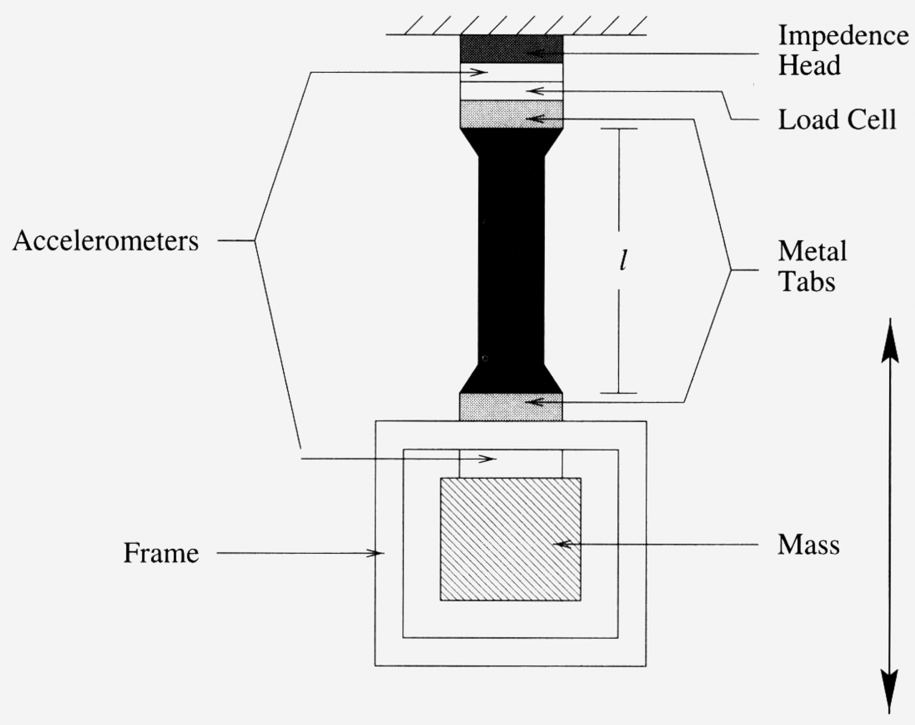

FIG. 2. Schematic of the experimental device for tensile validation tests

fixed $M=2$ in (4.5) with $p_{1}=p_{2}=1 / 2$. For highly filled rubber we found estimation with one uniform relaxation time $(M=1)$ would not adequately describe the data. The details for the case $M=2$ are given in $[7,10]$ and a typical comparison of the optimized fit model (i.e., model with estimated parameters) to data is depicted in Figure 3.

Partial theoretical foundations for such inverse problems are available [9] with a complete theory currently being pursued by the authors of this paper. As we noted above, in [1], well-posedness results (existence, uniqueness) for forward systems of the form (4.1), with various approximations of (4.3), and (4.5) are given under quite general assumptions on the nonlinearities. A careful formulation of the associated inverse problem for estimation of $\Phi$ in a class of probability measures $\mathcal{P}$ is given in [5] in the context of the Prohorov metric topology on $\mathcal{P}$. Computational approaches for problems similar to these are discussed in [4]. Our efforts to develop a complete theoretical as well as computational framework for inverse problems entailing (4.1), (4.3), (4.4) are underway.

Acknowledgments. This research was supported in part by the U.S. Air Force Office of Scientific Research under grants AFOSR F49620-01-1-0026 (HTB and NGM) and AFOSR F49620-03-1-0185 (GAP) and was facilitated through visits of the authors to the Statistical and Applied Mathematical Sciences Institute (SAMSI), which is funded by NSF under grant DMS-0112069. The first author (HTB) is grateful to Prof. J. Whiteman and the Brunel Institute of Computational Mathematics, Uxbridge, UK for the opportunity to participate in the "International Workshop on Viscoelastic Constitutive Modelling and Computation", June 25, 2003, which stimulated new directions for this research. 


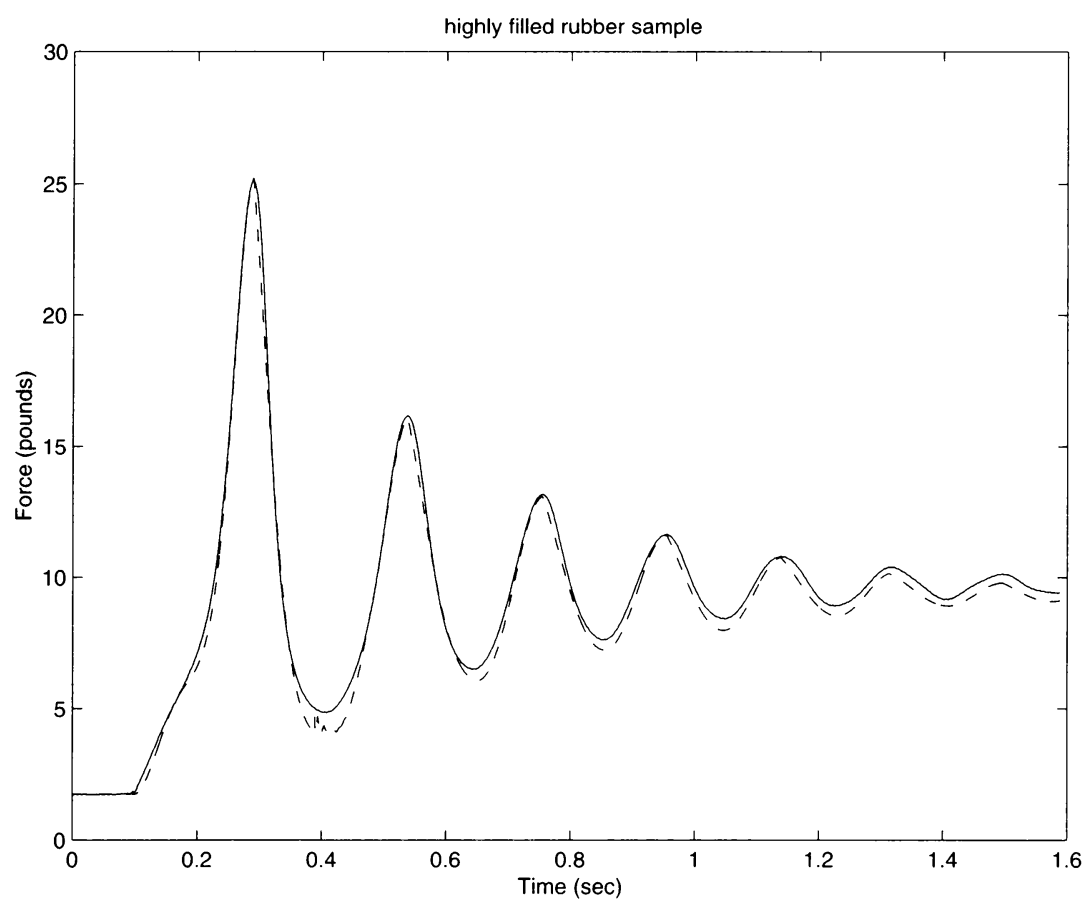

FIG. 3. Approximation with two internal variables (dashed line) and experimental data (solid line)

\section{REFERENCES}

[1] A.C. Ackleh, H.T. Banks and G.A. Pinter, Well-posedness results for models of elastomers, J. Math. Analysis and Applications 268 (2002), 440-456.

[2] R.D. Andrews, Correlation of dynamic and static measurements on rubberlike materials, Ind. Engr. Chem., 44 (1952), 707-715.

[3] H.T. Banks, J.H. Barnes, A. Eberhardt, H.T. Tran and S. Wynne, Modeling and computation of propagating waves from coronary stenoses, Comp. and Applied Math., 21 (2002), 1-22.

[4] H.T. Banks and K. Bihari, Modeling and estimating uncertainty in parameter estimation, CRSCTR99-40, NCSU, December 1999; Inverse Problems 17 (2001), 1-17.

[5] H.T. Banks, D. Bortz, G.A. Pinter and L.K. Potter, Modeling and imaging techniques with potential application in bioterrorism, CRSC-TR03-02, NCSU, January 2003; Chapter 6 in Bioterrorism: Mathematical Modeling Applications in Homeland Security, (H.T. Banks and C. Castillo-Chavez, eds.), Frontiers in Applied Mathematics, Vol.28, SIAM, Philadelphia, 2003, 129-154.

[6] H.T. Banks and N.G. Medhin, A molecular based dynamic model for viscoelastic responses of rubber in tensile deformations, CRSC-TR00-27, NCSU, October, 2000; Communications on Applied Nonlinear Analysis 8 (2001), 1-18.

[7] H.T. Banks, N.G. Medhin and G.A. Pinter, Multiscale considerations in modeling of nonlinear elastomers, CRSC-TR03-42, NCSU, October, 2003; J. Comp. Meth. Sci. Engr., to appear.

[8] H.T. Banks, H.T. Tran and S. Wynne, A well-posedness result for a shear wave propagation model, Intl. Series Num. Math., Vol.143, Birkhauser Verlag, Basel, 2002, 25-40.

[9] H.T. Banks and G.A. Pinter, Approximation results for parameter estimation in a class of abstract nonlinear hyperbolic systems, Appl. Math. Letters 12 (1999), 129-133.

[10] H.T. Banks, G.A. Pintér, L.K. Potter, M.J. Gaitens and L.C. Yanyo, Modeling of nonlinear hysteresis in elastomers under uniaxial tension, J. Intelligent Material Systems and Structures 10 (1999), 116-134. 
[11] G. Bishko, T.C.B. McLeish, O.G. Harlen and R.G. Larson, Theoretical molecular rheology of branched polymers in simple and complex flows: The pom-pom model, Phys. Rev. Lett., 79 (1997), 2352-2355.

[12] R. Blackwell, O.G. Harlen and T.C.B. McLeish, Theoretical linear and nonlinear rheology of symmetric treelike polymer melts, Macromolecules 34 (2001), 2579-2596.

[13] R. Blackwell, T.C.B. McLeish and O.G. Harlen, Molecular drag-strain coupling in branched polymer melts, J. Rheology 44 (2000), 121-136.

[14] C.J.F. Böttcher and P. Bordewijk, Theory of Electric Polarization, Vol.II: Dielectrics in Time Dependent Fields, Elsevier, Amsterdam, 1978.

[15] M. Doi and M. Edwards, The Theory of Polymer Dynamics, Oxford, New York, 1986.

[16] J.D. Ferry, E.R. Fitzgerald, L.D. Grandine and M.L. Williams, Temperature dependence of dynamic properties of elastomers: relaxation distributions, Ind. Engr. Chem., 44 (1952), 703706.

[17] Y.C. Fung, Biomechanics: Mechanical Properties of Living Tissues, Springer Verlag, New York, 1993.

[18] R.S. Graham, T.C.B. McLeish and O.G. Harlen, Using the pom-pom equations to analyze polymer melts in exponential shear, J. Rheology 45 (2001), 275-290.

[19] A.R. Johnson, C.J. Quigley and J.L. Mead, Large strain viscoelastic constitutive models for rubber, part I: Formulations, Rubber Chemistry Technology 67 (1994), 904-917.

[20] A.R. Johnson and R.G. Stacer, Rubber viscoelasticity using the physically constrained systems' stretches as internal variables, Rubber Chemistry Technology 66 (1993), 567-577.

[21] A.R. Johnson, C.J. Quigley, D.G. Young and J.A. Danik, Viscohyperelastic modeling of rubber vulcanization, Tire Sci. Tech., 21 (1993), 179-199.

[22] T.C.B. McLeish and R.G. Larson, Molecular constitutive equations for a class of branched polymers: The pom-pom polymer, J. Rheology 42 (1998), 81-110.

[23] F. Schwarzl and A.J. Staverman, Higher approximation methods for the relaxation spectrum from static and dynamic measurements of viscoelastic materials, Appl. Sci. Res., A4 (1953), 127-141.

[24] D. Ter Haar, A phenomenological theory of viscoelastic behavior, Physica 16 (1950), 839-850.

[25] E.R. Von Schweidler, Studien uber die anomalien im verhalten der dielektrika, Ann. Physik 24 (1907), 711-770.

[26] K.W. Wagner, Zur theorie der unvollkommenen dielektrika, Ann. Physik 40 (1913), 817-855.

[27] M.L. Williams and J.D. Ferry, Second approximation calculations of mechanical and electrical relaxation and retardation distributions, J. Poly. Sci., 11 (1953), 169-175. 